\title{
WHAT IS A SHELL-CROSSING SINGULARITY?
}

\author{
PETER SZEKERES ${ }^{1}$ and ANTHONY LUN ${ }^{2}$
}

(Received 27 February 1995)

\begin{abstract}
A detailed discussion of Newtonian and general relativistic spherically symmetric dust solutions leads to the following suggested criteria for a singularity to be classified as a shell-cross: (1) All Jacobi fields have finite limits (in an orthonormal parallel propagated frame) as they approach the singularity. (2) The boundary region forms an essential $C^{2}$ singularity which is $C^{1}$ regular, that is it can be transformed away by a $C^{1}$ coordinate transformation.
\end{abstract}

\section{Introduction}

Some proposed counterexamples to the cosmic censorship hypothesis $[8,12]$ have been discounted on the grounds that they are "shell-crossings" $[2,5]$. While this is undoubtedly a valid criticism in these particular cases, it does raise the general question: what exactly is a shell-crossing singularity? More specifically, given a singularity of a fluid collapse, what criteria should one use to label it a shell-cross rather than, say, a "central crush"?

We begin our discussion in this paper with a detailed examination of Newtonian spherically symmetric dust solutions. These solutions are essentially identical in all details with their general relativistic counterparts (the Tolman-Bondi solutions $[1,11])$, a feature which is perhaps not fully appreciated, yet there are none of the interpretational difficulties which occur in the latter. In Section 2 we show how to construct the general Newtonian solution by using Lagrangian coordinates. Taking the zero-energy case as a simple model, the difference between crushing and shellcrossing singularities is described. It is shown that in the case of a shell-cross the tidal forces are still infinite, but the integrated effect on a pair of neighbouring particles is finite, so that they are not compelled either to converge or diverge from each other.

\footnotetext{
${ }^{1}$ Department of Physics and Mathematical Physics, University of Adelaide, Adelaide SA 5005, Australia.

2Department of Mathematics, Monash University, Clayton, Victoria 3168, Australia.

(C) Australian Mathematical Society 1999, Serial-fee code 0334-2700/99
} 
In Section 3 we perform the analogous discussion for the Tolman-Bondi solutions. It is shown that the behaviour of the gravitational invariant $\Psi_{2}$ at a central crush is identical to that of a Schwarzschild solution, so that the matter content has essentially no effect on tidal effects there. The solutions are in this sense "vacuum dominated" at a central crush. Curiously this behaviour is completely mass-independent, giving it a kind of universality which is quite different to the behaviour at a shell-cross. The Jacobi equation and Newtonian tidal force equation derived in Section 2 are shown to be identical, so that the Newtonian discussion carries over unchanged to the general relativistic case.

In Section 4 we point out that the kind of tidal behaviour shown to hold at shellcrosses leads us to expect that the metric is not totally irregular there. We verify that for spherically symmetry it is possible to find coordinates such that the metric is $C^{\mathbf{l}}$ but not $C^{2}$ at the shell-cross singularity. In the final section we propose this as a general criterion for a singularity to be classified as a shell-cross and suggest a rewording of the cosmic censorship hypothesis.

\section{Newtonian theory}

Consider a spherically symmetric Newtonian pressure-free fluid of density $\rho=$ $\rho(r, t)$ and velocity field $\mathbf{v}=v(r, t) \mathbf{r}$ in a gravitational potential $\phi$. The Eulerian equations of motion are

$$
\begin{gathered}
\frac{\partial \rho}{\partial t}+\nabla \cdot(\rho \mathbf{v})=\frac{d \rho}{d t}+\frac{\rho}{r^{2}}\left(r^{2} v\right)^{\prime}=0, \\
\frac{d v}{d t}=\frac{\partial v}{\partial t}+v v^{\prime}=-\phi^{\prime},
\end{gathered}
$$

and

$$
\nabla^{2} \phi=\phi^{\prime \prime}+\frac{2}{r} \phi^{\prime}=4 \pi G \rho,
$$

where ${ }^{\prime} \equiv \partial / \partial r$. The Poisson equation (3) can be integrated to give

$$
\phi^{\prime}=\frac{G M_{r}}{r^{2}}
$$

where

$$
M_{r}(t)=\int_{0}^{r} 4 \pi \rho r^{2} d r
$$


To integrate this system it is best to use Lagrangian coordinates, $\mathbf{q}=$ the position which a particle at $(\mathbf{r}, t)$ occupies at time $t=0$. These coordinates evidently remain constant along the particle's motion and are the Newtonian analog of "comoving coordinates" in general relativity. Setting

$$
\mathbf{r}=\mathbf{q} R(q, t) \quad \text { where } \quad R(q, 0)=1 \quad \text { and } \quad q=\sqrt{\mathbf{q} \cdot \mathbf{q}}
$$

we have from $d \mathbf{q} / d t=\mathbf{0}$ that

$$
v=\left|\frac{d \mathbf{r}}{d t}\right|=R q \quad \text { and } \quad \frac{d v}{d t}=\left.\frac{\partial v}{\partial t}\right|_{q}=\ddot{R} q \quad\left(.\left.\equiv \frac{\partial}{\partial t}\right|_{q}\right) .
$$

Combining (2) and (4) gives

$$
\ddot{R}=-\frac{G M_{r}(t)}{R^{2} q^{3}} .
$$

The expansion parameter $\theta$ is given by

$$
\begin{aligned}
\theta=\nabla . \mathbf{v} & =\frac{2}{r} v+v^{\prime} \\
& =\frac{2 \dot{R}}{R}+\frac{1}{R+q R_{q}} \frac{\partial}{\partial q}(q \dot{R}) \\
& =\frac{\partial}{\partial t} \ln \left(R^{2}\left(R+q R_{q}\right)\right),
\end{aligned}
$$

where $R_{q}=\partial R / \partial q$, and the equation of continuity (1) integrates to give

$$
\rho=\frac{\rho_{0}(q)}{R^{2}\left(R+q R_{q}\right)} \text {. }
$$

Hence, from (5) we see, as expected intuitively from conservation of mass, that $M_{r}$ is a function of the Lagrangian radius $q$,

$$
M_{r}=\int_{0}^{q} 4 \pi q^{2} \rho_{0}(q) d q=M(q) .
$$

Equation (8) may now be integrated once to give the energy equation

$$
\dot{R}^{2}=\frac{2 G M(q)}{R q^{3}}+W(q)
$$

where $W(q)$ is an arbitrary function. $W$ can be thought of physically as representing the kinetic energy of the shell $q=$ constant at $R=\infty$. Equation (12) is integrable in elementary functions, being essentially a Friedmann equation, but by far the easiest 
case is $W(q)=0$ (the so-called "zero energy" case). Using the initial condition $R(q, 0)=1$, we find for this case

$$
R(q, t)=\left(1-\frac{t}{t_{0}(q)}\right)^{2 / 3},
$$

where

$$
t_{0}(q)=\sqrt{\frac{2 q^{3}}{9 G M(q)}} .
$$

As $t \rightarrow t_{0}$ we have $R \rightarrow 0$ and from (10) the density becomes infinite. The first term on the right hand side of (12) dominates the behaviour of all solutions in this limit, and there is little loss of generality in specializing to the case $W=0$. Using (11) we obtain

$$
\rho_{0}=\frac{1}{4 \pi q^{2}} M^{\prime}(q)=\frac{1}{6 \pi G t_{0}^{2}}\left(1-\frac{2 q}{3} \frac{t_{0}^{\prime}}{t_{0}}\right) \quad\left({ }^{\prime} \equiv \frac{d}{d q}\right),
$$

whence from (10)

$$
\rho=\frac{1}{6 \pi G\left(t_{0}-t\right)\left(t_{1}-t\right)},
$$

where

$$
t_{1}(q)=t_{0}(q)\left(1-\frac{2 q}{3} \frac{t_{0}^{\prime}}{t_{0}}\right)^{-1}
$$

There are thus two types of singularity where the density $\rho \rightarrow \infty$ :

1. As $t \rightarrow t_{0}(q)$, we have $R \rightarrow 0$ and the radius $r=R q$ of the sphere $q=$ constant approaches zero while the velocity $v=\dot{R} q \rightarrow \infty$. This is called a central crush.

2. As $t \rightarrow t_{1}(q)$ on the other hand, $R \rightarrow$ a finite value, so that $r=R q$ and $v$ both approach finite values. This much milder type of singularity is called a shell-cross. It is essentially independent of gravity, being the place where one shell of matter overtakes another one.

Consider now the tidal forces at the two types of singularity. The Newtonian tidal equation is

$$
\delta \ddot{x}_{i}=-\phi_{. i j} \delta x_{j} .
$$

Using the Poisson equation (3) it is not difficult to break $\phi_{, i j}$ into a matter part and a trace-free pure gravitational (Weyl) part,

$$
\phi_{. i j}=\frac{4}{3} \pi G \rho \delta_{i j}+\psi_{i j},
$$


where

$$
\psi_{i j}=\left(\phi^{\prime \prime}-\frac{\phi^{\prime}}{r}\right)\left(n_{i} n_{j}-\frac{1}{3} \delta_{i j}\right), \quad\left(n_{i}=\frac{x_{i}}{r}\right) .
$$

For the zero-energy case we find, using (4), (13), (14) and (15), that

$$
\psi_{i j}=\frac{2}{3} \frac{t_{0}-t_{1}}{\left(t_{0}-t\right)^{2}\left(t_{1}-t\right)}\left(n_{i} n_{j}-\frac{1}{3} \delta_{i j}\right)
$$

and

$$
\phi_{. i j}=\frac{2}{3} \frac{t_{0}-t_{1}}{\left(t_{0}-t\right)^{2}\left(t_{1}-t\right)} n_{i} n_{j}+\frac{2}{9\left(t_{0}-t\right)^{2}} \delta_{i j} .
$$

Hence separations $\delta x_{2}$ and $\delta x_{3}$ which are transverse to the radial direction $\mathbf{n}$ are subject to the equation

$$
\delta \ddot{x}+\frac{2}{9\left(t_{0}-t\right)^{2}} \delta x=0
$$

which has the solution

$$
\delta x=\delta_{0}\left(t_{0}-t\right)^{1 / 3}+\delta_{1}\left(t_{0}-t\right)^{2 / 3} \rightarrow 0, \quad\left(\delta_{0}, \delta_{1}=\text { consts }\right)
$$

while radial separations $\delta x_{1}$ are governed by the equation

$$
\delta \ddot{x}_{1}+\frac{2}{9} \frac{\left(3 t_{0}-2 t_{1}-t\right)}{\left(t_{0}-t\right)^{2}\left(t_{1}-t\right)} \delta x_{1}=0 .
$$

As $t \rightarrow t_{0}(q)$ this equation becomes approximately

$$
\delta \ddot{x}_{1} \approx \frac{4}{9} \frac{\delta x_{1}}{\left(t_{0}-t\right)^{2}}
$$

and has the asymptotic solution

$$
\delta x_{1} \approx \delta_{0}\left(t_{0}-t\right)^{4 / 3}+\delta_{1}\left(t_{0}-t\right)^{-1 / 3} .
$$

Although the divergent $\delta_{1}$ term dominates the radial behaviour a generic volume $\delta x_{1} \delta x_{2} \delta x_{3} \propto\left(t_{0}-t\right)^{1 / 3}$ will still tend to zero, which is another good reason for calling this a "crushing singularity".

Contrast this with the behaviour as $t \rightarrow t_{1}(q)$,

$$
\begin{aligned}
& \delta \ddot{x}_{1} \approx-\frac{2}{3\left(t_{0}-t_{1}\right)\left(t_{1}-t\right)} \delta x_{1}, \\
& \delta \ddot{x}_{i} \approx-\frac{2}{9\left(t_{0}-t_{1}\right)^{2}} \delta x_{i} \quad(i=2,3) .
\end{aligned}
$$


Transverse separations satisfy a simple harmonic oscillator equation and remain bounded and non-zero in general as $t \rightarrow t_{1}$, while radial separations behave as

$$
\delta x_{1} \approx\left(t_{1}-t\right)^{1 / 2}\left(\delta_{0} J_{1}\left(2 \sqrt{\alpha\left(t_{1}-t\right)}\right)+\delta_{1} Y_{1}\left(2 \sqrt{\alpha\left(t_{1}-t\right)}\right)\right),
$$

where $\alpha=2 /\left(3\left(t_{0}-t_{1}\right)\right)$ and $J_{1}$ and $Y_{1}$ are standard Bessel functions. The series expression for this solution is

$$
\begin{aligned}
\delta x_{1} \approx & \delta_{0}^{\prime}\left[\left(t_{1}-t\right)-\frac{1}{2} \alpha\left(t_{1}-t\right)^{2}+\cdots\right]+\delta_{1}^{\prime}\left[1-\frac{3}{4} \alpha^{2}\left(t_{1}-t\right)^{2}\right. \\
& \left.+\cdots+\ln \left(t_{1}-t\right)\left(-\alpha\left(t_{1}-t\right)+\frac{1}{2} \alpha^{2}\left(t_{1}-t\right)^{2}+\cdots\right)\right]
\end{aligned}
$$

which also has a finite value as $t \rightarrow t_{1}$. Thus while tidal forces become infinite as $t \rightarrow t_{1}$ they do so in a rather weak fashion, neighbouring particles in general remaining separated. Of course this is not true for particles on two neighboring shells, since the shells do cross at $t=t_{1}$ - such particles presumably correspond to the solution $\delta_{1}^{\prime}=0$ of the $\delta x_{1}$ equation.

\section{Tolman-Bondi collapses}

Consider now the well-known solutions in general relativity for a spherically symmetric collapsing dust cloud $[1,11]$,

$$
d s^{2}=-d t^{2}+\frac{S^{2}}{1+E(r)} d r^{2}+S^{2}\left(d \theta^{2}+\sin ^{2} \theta d \phi^{2}\right)
$$

where' $\equiv \partial / \partial r, \cdot \equiv \partial / \partial t$ and $S=S(r, t)$ satisfies a "Friedmann" equation which, with an appropriate choice of radial coordinate $r$, can be made to take the form

$$
\dot{S}^{2}=E(r)+\frac{4 r^{3}}{9 S}
$$

The energy-stress tensor is

$$
T_{\mu \nu}=\frac{1}{8 \pi G} G_{\mu \nu}=\rho u_{\mu} u_{\nu},
$$

where $u^{\mu}=(1,0,0,0)$ and the density $\rho$ is given by

$$
\rho=\frac{r^{2}}{6 \pi G S^{2} S^{\prime}}
$$


The function $E(r)$ may be arbitrarily specified, subject to the constraint $E(r)>-1$. It is a measure of the energy of a shell of radius $r$. To understand this, note that any such shell has "physical" radius $S$ as given by the area formula $A=4 \pi S^{2}$, and whenever $E \geq 0$ we have $E \approx \dot{S}^{2}$ as $S \rightarrow \infty$. Arguing by analogy, the case $E<0$ is given the same interpretation.

These solutions correspond precisely to the Newtonian solution discussed in Section 2 if we regard $S$ as being identified with the Euclidean radial coordinate $r$. If we set, in analogy with the previous section,

$$
q=S(r, 0) \quad \text { and } \quad S=R(q, t) q,
$$

so that $R(q, 0)=1$, then (25) and (27) agree exactly with (12) and (10) provided we make the identifications

$$
W(q)=\frac{E(r)}{q^{2}}, \quad M(q)=\frac{2 r^{3}}{9 G}, \quad \text { and } \quad \rho_{0}=\frac{r^{2}}{6 \pi G q^{2}} .
$$

The only deficiency with this association of the two solutions is that the actual mass $\bar{M}_{r}$ within a sphere of radius $r$ is given in general relativity by

$$
\bar{M}_{r}=\int_{0}^{\infty} \frac{4 \pi S^{2} S^{\prime} \rho}{\sqrt{1+E(r)}} d r=\frac{2}{3 G} \int_{0}^{\infty} \frac{r^{2} d r}{\sqrt{1+E(r)}},
$$

which only agrees with the Newtonian $M_{r}$ for the zero-energy case $E(r)=0$.

For the simple zero-energy case $E(r)=0$, where each shell just comes to rest at infinity, (25) integrates to

$$
S=r\left(t_{0}(r)-t\right)^{2 / 3},
$$

where $t_{0}(r)$ is an arbitrary function. From (27) the density in this case is

$$
\rho=\frac{1}{6 \pi G\left(t_{0}-t\right)\left(t_{1}-t\right)},
$$

where

$$
t_{1}(r)=t_{0}(r)+\frac{2}{3} r t_{0}^{\prime}(r)
$$

Note the slight difference between this and (16), although the two formulae agree asymptotically in the limit $\frac{t_{0}^{\prime}}{t_{0}} q \ll 1$. The density again becomes singular at both $t=t_{0}(r)$ and $t=t_{1}(r)$, the former being interpreted as a "central crush" since from (28) the area $4 \pi S^{2}$ vanishes there, while the latter has finite area in general and is called a "shell-cross". 
For the general case $E(r) \neq 0$ the situation is essentially identical. From (27) the density becomes infinite at $S=0$ and $S^{\prime}=0$. From the Friedmann (25) the behaviour near $S=0$ is dominated by the solution for $E=0$, so that $S$ is asymptotically equal to the form given in (28). Thus $S=0$ is a central crush singularity similar to $t=t_{0}(r)$ in the $E=0$ case, while $S^{\prime}=0$ is to be interpreted as a shell cross as in $t=t_{1}(r)$. There is therefore no loss of generality in continuing to discuss the $E=0$ case.

While there are two distinct types of singularity in these solutions, one should note however that, from (29), the density approaches infinity in identical ways at $t=t_{0}$ and $t=t_{1}$. Invariants of the Riemann tensor also become infinite at both types of singularity (this is obvious for the Ricci scalar which is proportional to $\rho$, but is true of invariants associated with the Weyl tensor as well). Always associated with such a curvature singularity will be infinite tidal forces.

How then do we distinguish between these two types of singularity? A clue can be obtained by computing the Weyl tensor. Since spherically symmetric metrics are of Petrov type D, only the Newman-Penrose invariant $\Psi_{2}$ need be computed [3]. For the zero-energy Tolman-Bondi metric this calculates to

$$
\Psi_{2}=-\frac{4 r t_{0}^{\prime}(r)}{27\left(t_{0}(r)-t\right)^{2}\left(t_{1}(r)-t\right)} .
$$

Thus $\Psi_{2}$ approaches infinity both as $t \rightarrow t_{0}(r)$ and as $t \rightarrow t_{1}(r)$, but the power law behaviour is different at these two limits, being much more rapid at the central crush $t \rightarrow t_{0}(r)$. The situation for more general Tolman-Bondi metrics $(E(r) \neq 0)$ can be shown to be quite similar, the invariant $\Psi_{2} \rightarrow \infty$ both as $S(r, t) \rightarrow 0$ and $S^{\prime}(r, t) \rightarrow 0$, but in the former case it behaves as the inverse square of the proper time, while in the latter (shell-cross) it varies only as the inverse power of the proper time.

It is interesting to compute $\Psi_{2}$ for the Schwarzschild solution of mass $m$, which in comoving coordinates takes the form [7]

$$
d s^{2}=-d t^{2}+\frac{4}{9} A^{2}\left(t_{0}(r)-t\right)^{-2 / 3} t_{0}^{\prime}(r) d r^{2}+A^{2}\left(t_{0}(r)-t\right)^{4 / 3}\left(d \theta^{2}+\sin ^{2} \theta \phi^{2}\right),
$$

where $A=(9 m / 2)^{1 / 3}$. The relation these coordinates have with standard Schwarzschild coordinates $\bar{r}$ and $\bar{t}$ is

$$
\begin{aligned}
& \bar{r}=A\left(t_{0}(r)-t\right)^{2 / 3}, \\
& \bar{t}=-t+(8 m \bar{r})^{1 / 2}+2 m \ln \frac{1-(2 m / \bar{r})^{1 / 2}}{1+(2 m / \bar{r})^{1 / 2}} .
\end{aligned}
$$

The reader may readily check that the metric (32) transforms to the usual Schwarzschild form

$$
d s^{2}=-\left(1-\frac{2 m}{\bar{r}}\right) d \bar{t}^{2}+\frac{d \bar{r}^{2}}{1-(2 m / \bar{r})}+\bar{r}^{2}\left(d \theta^{2}+\sin ^{2} \theta d \phi^{2}\right),
$$


and the invariant $\Psi_{2}$ has the value

$$
\Psi_{2}=-\frac{m}{\bar{r}^{3}}=-\frac{2}{9\left(t_{0}(r)-t\right)^{2}}
$$

It is remarkable that the $\Psi_{2}$ exhibits a kind of universal behaviour, in that it grows at a rate independent of the mass of the Schwarzschild particle or of the initial velocity of the observer.

From (30) and (31) we see that the behaviour of $\Psi_{2}$ in a central crush is identical to the universal Schwarzschild behaviour, so the singularity can be thought of as being "vacuum dominated" [9].

To understand the fundamental difference between this vacuum type behaviour and the shell-cross case $\left(t=t_{1}(r)\right)$ we consider the equation of geodesic deviation which defines a Jacobi field $\eta^{\mu}$. In an orthonormal frame $e_{(i)}^{\mu}(i=1,2,3)$ and $e_{(4)}^{\mu}=u^{\mu}$ this equation has the form

$$
\ddot{\eta}_{i}=-\sum_{j} R_{i 4 j 4} \eta_{j}
$$

where

$$
R_{i 4 j 4}=R_{\mu \nu \rho \sigma} e_{(i)}^{\mu} e_{(4)}^{\nu} e_{(j)}^{\rho} e_{(4)}^{\sigma} .
$$

In the zero-energy case

$$
\begin{aligned}
& R_{1414}=R_{r t r t}\left(t_{0}-t\right)^{2 / 3}\left(t_{1}-t\right)^{-2}=\frac{2}{9} \frac{\left(3 t_{0}-2 t_{1}-t\right)}{\left(t_{0}-t\right)^{2}\left(t_{1}-t\right)}, \\
& R_{2424}=R_{\theta t \theta t} r^{-2}\left(t_{0}-t\right)^{4 / 3}=\frac{2}{9\left(t_{0}-t\right)^{2}}
\end{aligned}
$$

and from (17), (22) and (23) we see that

$$
R_{1414}=\phi_{.11} \quad \text { and } \quad R_{2424}=\phi_{, 22}
$$

Hence the Jacobi equation (34) and the Newtonian tidal equation (17) are completely identical in the zero-energy case. This completes the correspondence between the Tolman-Bondi metrics and their Newtonian counterparts. The whole of the discussion in the previous section, making clear the difference between the vacuum dominated $t_{0}$ singularity and the shell-cross case $t_{1}$ can therefore be applied to the general relativistic case as it stands. 


\section{Regularization of the metric at a shell-cross.}

The behaviour at a central crush (for example, $t=t_{0}(r)$ in the zero-energy case) is asymptotically similar to the universal Schwarzschild behaviour at $\bar{r}=0$, which is well known to be an irremovable singularity. While the shell-cross singularity at $t=t_{1}$ also has infinite curvature invariants, the situation from a tidal or Jacobi field point of view is much weaker, as we have seen above. We shall now show that in a sense it is a removable singularity.

Consider a metric of the form

$$
d s^{2}=-d t^{2}+(\tau(r)-t)^{2} F^{2}(r, t) d r^{2}+G^{2}(r, t)\left(d \theta^{2}+\sin ^{2} \theta d \phi^{2}\right),
$$

where $F$ and $G$ are functions which are regular at $t=\tau(r)$. The zero-energy TolmanBondi metric

$$
d s^{2}=-d t^{2}+\left(t_{0}-t\right)^{-2 / 3}\left(t_{1}-t\right)^{2} d r^{2}+r^{2}\left(t_{0}-t\right)^{4 / 3}\left(d \theta^{2}+\sin ^{2} \theta d \phi^{2}\right),
$$

where

$$
t_{1}(r)=t_{0}(r)+\frac{2}{3} r t_{0}^{\prime}(r)
$$

is clearly of this form at $t=t_{1}(r)$ wherever $t_{0}^{\prime}(r) \neq 0$, on setting $\tau(r)=t_{1}(r)$, $F(r, t)=\left(t_{0}(r)-t\right)^{-1 / 3}$ and $G(r, t)=r\left(t_{0}(r)-t\right)^{2 / 3}$.

The hint that such metrics might be regular is found from the 2-dimensional Rindler space

$$
d s^{2}=-d t^{2}+t^{2} d r^{2}
$$

which reduces to Minkowski space $d s^{2}=-d T^{2}+d R^{2}$ if we put $r=\operatorname{arctanh}(T / R)$ and $t=\sqrt{T^{2}-R^{2}}$. Adopting a recent proposal of Szekeres and Iyer [10] we try a transformation to coordinates $(u, x)$ of the form

$$
\begin{aligned}
& t=\tau(u)-a_{2}(u) x-a_{3}(u) x^{3 / 2} \\
& r=u+b_{1}(u) x^{1 / 2}+b_{2}(u) x+b_{3}(u) x^{3 / 2} .
\end{aligned}
$$

It is clear that $x=0$ corresponds to the singularity $t=\tau(r)$ of the metric (35), and that we may expand the function $\tau(r)$ in terms of $u$ and $x$,

$$
\tau(r)=\tau(u)+b_{1} \tau^{\prime}(u) x^{1 / 2}+\left(\frac{1}{2} b_{1}^{2} \tau^{\prime \prime}(u)+b_{2} \tau^{\prime}(u)\right) x+O\left(x^{3 / 2}\right),
$$

where ${ }^{\prime} \equiv d / d u$. Expanding the function $F$ as a series in $u$ and $x^{1 / 2}$

$$
F=\phi_{0}(u)+\phi_{1}(u) x^{1 / 2}+\phi_{2}(u) x+\cdots
$$


we find that the metric components in $(u, x)$ coordinates have the expansion

$$
\begin{aligned}
g_{u u}= & -\left(\tau^{\prime}\right)^{2}+\tau^{\prime}\left(2 a_{2}^{\prime}+b_{1}^{2} \tau^{\prime} \phi_{0}^{2}\right) x+O\left(x^{3 / 2}\right), \\
g_{u x}= & a_{2} \tau^{\prime}+\frac{\tau^{\prime}}{2}\left(3 a_{3}+b_{1}^{3} \tau^{\prime} \phi_{0}^{2}\right) x^{1 / 2}+O(x)+O\left(x^{3 / 2}\right), \\
g_{x x}= & -a_{2}^{2}+\frac{1}{4} b_{1}^{4} \tau^{\prime 2} \phi_{0}^{2} \\
& +\left(-3 a_{2} a_{3}+\frac{1}{4} b_{1}^{3} \tau^{\prime} \phi_{0}\left(6 \tau^{\prime} \phi_{0} b_{2}+2 b_{1} \tau^{\prime} \phi_{1}+b_{1}^{2} \phi_{0} \tau^{\prime \prime}+2 \phi_{0} a_{2}\right)\right) x^{1 / 2} \\
& +O(x)+O\left(x^{3 / 2}\right) .
\end{aligned}
$$

It is possible that all coefficients of $x^{1 / 2}$ in these series vanish if we set

$$
\begin{aligned}
& b_{1}=\phi_{0}^{-1 / 2}, \\
& a_{2}=\frac{1}{2} \tau^{\prime}, \\
& a_{3}=-\frac{1}{3} \tau^{\prime} \phi_{0}^{1 / 2} \\
& b_{2}=-\frac{1}{2}-\frac{\phi_{1}}{3 \phi_{0}^{3 / 2}}-\frac{\tau^{\prime \prime}}{6 \phi_{0} \tau^{\prime}} .
\end{aligned}
$$

The terms $b_{1}$ and $a_{2}$ can be defined arbitrarily, but the above choices have been adopted for reasons of simplicity. The metric components $g_{u u}, g_{u x}$ and $g_{x x}$ are all $C^{1}$ functions of $x$, but $g_{22}=G(r, t)$ will still have $x^{1 / 2}$ terms in its expansion in general. In the case of a shell-cross singularity however this will not be so, for if we expand $t_{0}(r)-r$ in terms of $u$ and $x$ we find, on using

$$
\tau(u)=t_{1}(u)=t_{0}(u)+\frac{2}{3} u t_{0}^{\prime}(u)
$$

that

$$
t_{0}(r)-t=-\frac{2}{3} u t_{0}^{\prime}(u)+b_{1}(u) t_{0}^{\prime}(u) x^{1 / 2}+O(x)+O\left(x^{3 / 2}\right),
$$

whence

$$
G(r, t)=r\left(t_{0}(r)-t\right)^{2 / 3}=u\left(-\frac{2}{3} u t_{0}^{\prime}(u)\right)^{2 / 3}\left(1+O(x)+O\left(x^{3 / 2}\right)\right)
$$

and

$$
F(r, t)=\left(t_{0}(r)-t\right)^{-1 / 3}=\left(-\frac{2}{3} u t_{0}^{\prime}(u)\right)^{-1 / 3}\left(1+\frac{b_{1}(u)}{2 u} x^{1 / 2}+O(x)\right),
$$


where

$$
\phi_{0}(u)=\left(-\frac{2}{3} u t_{0}(u)\right)^{-1 / 3}, \quad \phi_{1}(u)=\frac{b_{1}(u)}{2 u} \phi_{0}(u)=\frac{\phi_{0}^{1 / 2}}{2 u} .
$$

Since $G(u, x)$ is clearly a $C^{1}$ function of $u$ and $x$, the whole metric is $C^{1}$. The coordinate transformation which has achieved this regularization of the shell-cross is

$$
\begin{aligned}
& t=t_{1}(u)-\frac{1}{2} t_{1}^{\prime}(u) x+\frac{1}{3} t_{1}^{\prime}(u) \phi_{0}^{1 / 2} x^{3 / 2}, \\
& r=u+\phi_{0}^{-1 / 2} x^{1 / 2}-\frac{1}{6}\left(3+\frac{1}{u \phi_{0}(u)}+\frac{\tau^{\prime \prime}(u)}{\phi_{0}^{1 / 2}(u)}\right) x,
\end{aligned}
$$

where $\phi_{0}$ and $\phi_{1}$ are given by (46). In the language of the abstract boundary [6], the singularity at $t=t_{0}$ is $C^{1}$ essential while the shell-cross at $t=t_{1}$ is $C^{1}$ removable (can be covered by set of $C^{1}$ regular boundary points) but is $C^{2}$ essential. Similar conclusions were drawn quite a few years ago by Papapetrou and Hamoui, but only for a very specific Tolman-Bondi metric [4].

\section{Conclusions}

Two criteria present themselves as possible candidates for deciding whether a singularity in general relativity is a shell-cross:

1. If $\eta^{\mu}$ is a Jacobi field along any timelike geodesic which approaches the singularity with proper time parameter $t \rightarrow t_{1}$, then its components in a parallel propagated orthonormal basis have finite non-zero limit as $t \rightarrow t_{1}$.

2. A singularity of a collapsing matter solution in general relativity is a shell cross if it can be covered by a 3-surface of $C^{1}$ regular boundary points (see [6] for all relevant definitions). That is, if it is a $C^{2}$ essential singularity which is removable by a $C^{1}$ coordinate transformation.

It is not clear whether these two definitions are completely equivalent, but the discussion in this paper would indicate that there is a close link between the two criterion. While our preference is to regard Condition 2 as the most general criterion, there are practical difficulties in seeking coordinate transformations such as those discussed in Section 4. Hence it may be generally best to use Condition 1 as a calculational guide since it is usually much easier to apply.

Finally, it may be useful to formulate a statement of the cosmic censorship hypothesis, which excludes the case of shell-crossings, as these are often timelike and locally naked, but are not to be regarded as serious contenders for violating the hypothesis. We suggest the following statement of the strong cosmic censorship hypothesis: 
Given well-posed regular initial data on a space-like partial Cauchy surface $S$, the future Cauchy development of $S$ has no $C^{1}$ essentially singular abstract boundary point which is in the past of any regular point $p \in I^{+}(S)$.

What exactly constitutes "well-posed regular initial data" is deliberately left unspecified here. It would appear that inequalities such as those given by the dominant energy condition are not in themselves restrictive enough to ensure that the hypothesis is valid [10].

\section{Acknowledgment}

The authors would like to thank the referee for his careful reading of the manuscript and for pointing out several mistakes therein.

\section{References}

[1] H. Bondi, Mon. Not. Roy Astron. Soc. 107 (1947) 410.

[2] R. Geroch and G.T. Horowitz, in General Relativity: an Einstein Centenary Survey (eds. S. W. Hawking and W. Israel), (Cambridge University Press, 1979) 212.

[3] E. T. Newman and R. Penrose, "An approach to gravitational radiation by a method of spin coefficients", J. Math. Phys. 3 (1962) 566-578.

[4] A. Papapetrou and A. Hamoui, "Couches simples de matière en relativité générale", Ann. Inst. $H$. Poincaré 9 (1968) 179-211.

[5] R. Penrose, in General Relativity: an Einstein Centenary Survey (eds. S. W. Hawking and W. Israel), (Cambridge University Press, 1979) 581.

[6] S. M. Scott and P. Szekeres, "The abstract boundary - a new approach to singularities and manifolds", J. Geom. Phys. 13 (1994) 223-253.

[7] P. Szekeres, "Global description of spherical collapsing and expanding dust clouds", Il Nuovo Cimento 17B (1973) 187-195.

[8] P. Szekeres, “Quasispherical gravitational collapse”, Phys. Rev. D12 (1975) 2941-2948.

[9] P. Szekeres, in A Random Walk in Relativity and Cosmology: Essays in honour of P. C. Vaidya and A. K. Raychaudhuri (eds. N. Dadhich, J Krishna Rao, J. V. Narlikar and C. V. Vishveshvara), (Wiley Eastern Ltd, New Delhi, 1985) 211.

[10] P. Szekeres and V. Iyer, "Spherically symmetric singularities and strong cosmic censorship", Phys. Rev. D47 (1993) 4362-4371.

[11] R. C. Tolman, Proc. Nat. Acad. Sci. USA 20 (1934) 169.

[12] P. Yodzis, H-J. Seifert and H. Müller zum Hagen, "On the occurrence of naked singularities in general relativity", Commun. Math. Phys 34 (1973) 135-148. 